\title{
LE MALADE SE RANIME
}

\section{Bob Brown}

I should have remained burdened with destinations Perhaps, or stayed quite drunk, or obeyed The undertaker, who was fairly charming after all.

Weldon Kees

Une brise égale et différente soufflait entre les jambes de 1'agent qui se penchait et interpe1lait une masse couchée en chien de fusil sur le trottoir.

"Et vous, alors, on ne peut rien vous apprendre?"

Crosby essaya de se soulever loin de 1'humidité du macadam; il ne put que se mettre à genoux et décida alors de se laisser retomber, posant sa joue sur le sol frais et rassurant. A la radio du motard une voix douce murmura:

"Agent 40, Mike?"

L'agent s'avança vers Crosby, effleurant sa veste déchirée d'une botte noire bien cirée.

"36 rue des Noyers; ah! on dirait un essai de suicide; envoyez donc une ambulance."

"Tout de suite! Agent 40, restez sur place." Le motard obéissait au central et s'attardait 
auprès de Crosby jusqu'au moment où une ambulance émergeait du crépuscule. Ses lumières rouges éclairaient la devanture d'une boutique de poissons rouges en couchers de soleil accélérés et cosmiques. Crosby se terra au plus profond de lui-même, là où seule une pénombre bleue, fascinante, éclairée de diamants vert-tilleul restait pour lui; il s'éveilla quatre jours plus tard.

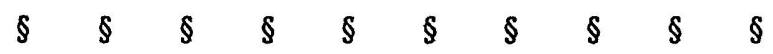

Quinze jours après, Crosby avait retrouvé toute sa connaissance et en même temps tous ses souvenirs et ses responsabilités. Il se décida à une trêve temporaire et signa les papiers qui donneraient à sa femme un divorce rapide. Le même jour, on 1ui


et il rencontra le docteur William Karst Amers qui insistait que tous ses clients 1 'appellent Karst.

Karst avait dit à Crosby qu'il avait 28 ans. I1 s'était spécialisé en psychiatrie après une dépression nerveuse subie pendant sa première année de médecine. I1 lui semblait que d'aider les autres pendant leurs accès de folie (non à les supprimer, en tout cas pas complètement) mettait de 1 'ordre dans ses propres idées de telle sorte qu'il pouvait agir de la manière dont les malades et autres pensaient qu'un psychiatre dut le faire. Crosby ne pensa guère de bien de Karst lors de leur première rencontre. En fait, il pensa que ce docteur Amers devrait peut-être prendre quelques vacances lui-même pour remettre ses propres billes en état de marche. Mais le divorce 1 'ayant dépossédé de sa boutique de poissons tropicaux, il n'avait plus rien d'autre à faire. Alors, il se décida à parler à Karst et ensuite à la secrétaire dé celui-ci. Deux mois plus tard, ou sept jeudis pour être plus précis, il emména- 
geait dans 1 'appartement de la secrétaire. Karst leur avait donné une télévision en coúleurs portative qui comprenait aussi une radio et un réveille-matin. La réceptionniste s'appelait Karen. Crosby ne pouvait se souvenir de son deuxième prénom, mais comme il 1'avait mentionné à Karst auparavant, elle avait des seins d'acier et un postérieur de nickel.

Avec elle comme avec les autres femmes, Crosby découvrit bientôt qu'ils avaient fort peu de choses à se dire. Il ne pensait pas que ce fût sa faute, et il essayait une fois par jour d'entrer en conversation avec Karen.

"Aimes-tu ton travail?"

Il avait posé la question journalière; il ouvrit alors une bière et s'installa sur le sofa zébré d'orange que la mère de Karen leur avait offert. Il attendit une réponse.

Karen, elle, aspirait la dernière bouffée d'une cigarette de Marie-Jeanne. Ses paupières s'ouvrirent légèrement, et elle considéra Crosby comme une punaise écrasée. Elle mouilla le bout de ses doigts, éteignit le feu, et avala le mégot.

"Crosby, je suis aussi entichée de mon boulot que $j e$ le suis de mes règles. Ils existent, un point c'est tout, et je saigne autant pour I'un que pour les autres. Passe-moi ta bière, chéri."

Crosby se pencha vers sa chaise et lui offrit son verre.

"Scatologiquement s'entend, n'est-ce pas?"

"Ne me fatigue pas avec tes histoires sarcastiques de dépression ou je vais raconter tout cela à Karst demain."

Crosby arracha son verre de bière à Karen et sourit.

"Demain, c'est dimanche."

Karen lui sourit à son tour.

"Crosby, pourquoi ne pouvons-nous jamais nous parler -- je veux dire réellement converser sur quelque chose?" 
Ses yeux s'agrandirent de déception comme si elle connaissait le jeu aussi bien que lui.

Parler n'intéressait plus Crosby, et finalement tout dialogue entre eux devint seulement une simple musique, sorte de toile de fond. Il arrêta même de faire sa promenade quotidienne dans le royaume de 1 a conversation et $n$ 'en fut pas plus malheureux pour cela. Il venait de trouver un nouvel objet d'intérêt. Il construisait un modèle réduit d'avion chaque jour. En éparpillant avec soin la colle forte et les pièces détachées, en arrangeant fidèlement les bombes et les mitrailleuses selon les stipulations du schéma, il pouvait occuper complètement ses journées.

I1 conduisait Karen à son bureau. Là il prenait avec Karst une tasse de café pendant que Karen mettait à jour les dossiers des consultations de la veille. Elle entrait et sortait, interrompant leur discussion sur les progrès mentaux de Crosby, s'arrêtait parfois pour siroter son café, intervenait pour placer une remarque sur un événement que Crosby ne voulait pas discuter avec son médecin. C'était thérapeutique pour les trois. Alors Crosby les laissait à leurs routines, leurs névroses, et leurs malades, et il gagnait le rayon des jouets de Korbettes. Là sa journée allait vraiment commencer. Ce magasin avait neuf cents modèles différents. Crosby ne s'occupait que de la Deuxième Guerre Mondiale et se limitait à environ cent quatre-vingt avions alliés ou allemands.

"Vous prenez le ME109 aujourd'hui, Monsieur?"

"Oui, je crois. Le camouflage pour la guerre dans le désert m'intéresse."

"Un peu d'authenticité, hein, Monsieur?"

"Mais oui, c'est cela, j'ai du reste un endroit spécial pour cet avion."

Heureux et pas du tout ennuyé par la 
familiarité du vendeur, Crosby rentra chez lui. I1 voulait passer la journée entière à travailler sur cet avion. Il était à l'échelle d'un vingthuitième et le resta jusque vers quatre heures de I'après-midi quand il commença le travail le plus intéressant, celui de peindre le visage du pilote. Il disposa ses couleurs, surtout des bleus, des verts, et un petit flacon d'un rose lumineux. II prit la photographie de son ex-femme dans la poche secrète de son portefeuille. I1 décalqua son visage sur le pilote en plastique, muet et immobile, cimenté dans le Messerschmitt. Il se rappela la dernière fois où il 1 'avait vue, le jeune homme et elle ensemble, dans la petite voiture de sport verte qu'il lui avait achetée. Et lui, regagnant sa boutique en voiture, et avalant les cachets. Les absorbant pour éviter de penser à toutes les possibilités entre elle et le garçon. Avec soin, il peignit une paire de sourcils verts au pilote et, choisissant un jaune d'or très proche de la couleur des cheveux de sa femme, il transforma le petit casque du pilote en une masse fluide de boucles blondes. Et il se souvenait des possibilités de mort 1 'effort désespéré pour sortir du magasin et s'écrouler sur le trottoir. Quelles possibilités pourrait-il envisager maintenant, peut-être un rose lumineux pour les lèvres? Oui, et les verres de contact verdâtres qu'elle affectionnait.

I1 se renversa dans sa chaise, alluma une cigarette, et envoya la fumée vers $I^{\prime}$ avion de chasse vert et brun. La fumée s'enroula en nuages autour des ailes, et la minuscule figure brillante dans le cockpit fut obscurcie par les émanations quand il ferma brusquement le poste de pilotage et s'éloigna sur le balcon. $\mathrm{Au}$ dessus, dans la cour, un groupe de jeunes locataires jouait au volleyball. Légèrement en arrière du groupe, deux femmes 
paressaient au bord de la piscine. Dans le soleil de 1'après-midi, les peaux bien huilées brillaient comme le dos d'un mocassin d'eau qu'il avait vu autrefois glisser d'un tronc d'arbre. Mille images vieillies lui revenaient, ni agréables ni déplaisantes, émergeant seulement de son passé. Elles dansaient dans son esprit et disparaissaient alors qu'il regardait 1 e jeu en dessous de lui.

Une des jeunes joueuses lui fit signe.

"Hé, descendez donc, descendez, voulez-vous?"

Il jeta sa cigarette dans le brasero et se pencha au-dessus de la balustrade.

"Hê, là-bas, qu'est-ce que vous voulez?"

La jeune fille dans un bikini à pois violemment coloré mit ses mains sur les hanches, face au balcon. Ses cheveux pendillaient en mèches mouillés.

"Descendez, venez vite, nous avons besoin d'un partenaire dans notre camp."

Se retournant vers le filet, elle ajouta,

"Pressez-vous, ils sont en train de nous battre."

"Une minute, je suis à vous."

II se précipita à l'intérieur de la chambre, arracha ses vêtements et mit une paire de nouveaux shorts de tennis. Il allait descendre quatre à quatre les escaliers quand il s'arrêta, rentra dans 1 'appartement et se changea, mettant cette fois-ci une paire de vieux jeans coupés aux genoux. En une minute il se retrouva sur $I^{\prime}$ herbe frâtche au milieu de corps jeunes et en sueur. La jeune fille au bikini lui envoya un grand sourire amical. Il dansait de place en place, claquant le ballon avec joie, prenant plaisir à la douleur cuisante, brève et excitante, les cris d'encouragement, les plaisanteries et risées quand il envoyait le ballon hors du jeu. Son équipe gagna.

II invita la jeune fille à monter boire une bière et 1 'entendit avec plaisir répondre: 
"Bien sûr, pourquoi pas?"

Quand ils entrèrent dans 1'appartement, Karst et Karen inspectaient I'avion. Les sourcils de Karen s'élévèrent de surprise, et Karst dit d'un ton de badinerie suave:

"Ah, bonjour! Crosby mon ami!"

Crosby les abandonna tous les trois dans une attente tranquille et revint avec une bière pour la jeune fille et une pour lui. Il fit sauter le bouchon; tout en guidant la jeune fille blonde hors de l'appartment, il se retourna sur le seuil, hocha la tête dans la direction de Karst et du ME109, puis regarda Karen qui cligna les yeux:

"C'est une beauté, n'est-ce pas?"

Il descendit et sortit dans le chaud soleil californien et demanda à la jeune fille:

"Je pourrais peut-être rester un jour ou deux chez vous?"

Elle avala une longue gorgée de bière et secoua les épaules.

"Bien sûr, pourquoi pas?"

La joie qu'il avait éprouvée pendant le match de volley s'évanouissait déjà alors qu'il suivait la jeune fille le long du corridor étroit et impersonnel. Il ne faisait guère attention à la chevelure sensuelle et humide qui caressait ses épaules mais notait seulement le grain veiné de sa peau et la masse veloutée du duvet blond à la base de son dos, juste au-dessus des hanches. Elle chantait doucement une mélopée qu'il ne pouvait saisir; il lui serra fermement le bras et demanda,

"Hé! Vous ne prenez rien, n'est-ce pas?"

Son chant s'arrêta. Dans le silence de son regard, il se sentit nerveux. Quand elle commença à parler, il n'entendit pas les mots mais vit seulement son visage et ses lèvres de poupée. Qu'est-ce qui ne marchait pas soudain? Il était fatigué de la pantomine, mais il ne savait que changer; il ne trouva rien à briser autour de lui. 
Il se mit alors à sourire comme s'il était un des visages d'une affiche de Coca-Cola. Il espérait que sa vitalité artificielle ne disparaîtrait pas trop tôt. Peut-être aujourd'hui ne serait-il pas plus intéressant qu'hier.

Wichita State University 